\title{
V355 And: a neglected detached binary in a multiple star system
}

\author{
B. Özkardeş ${ }^{1,2}$ \\ 1 Department of Space Science and Technologies, Faculty of Arts and \\ Sciences, Canakkale Onsekiz Mart University, Terzioğlu Kampüsü, \\ TR-17020, Canakkale,Turkey, (E-mail: burcu@comu.edu.tr) \\ 2 Canakkale Onsekiz Mart University, Astrophysics Research Center and \\ Ulupinar Observatory, TR-17100, Çanakkale, Turkey
}

Received: October 9, 2020; Accepted: December 28, 2020

\begin{abstract}
The analysis of light curves (from TESS, ASAS-SN and KWS databases) of the eclipsing binary V355 And is presented for the first time. The $O-C$ diagram was constructed using all available times of minimum light together with the ones determined in this study, and the revised ephemeris was obtained. The final photometric model describes V355 And as an Algollike type binary star with a detached configuration. Absolute parameters of the components of the system were found to be: masses, $M_{1}=1.56 \pm 0.01 \mathrm{M}_{\odot}$ and $M_{2}=1.38 \pm 0.01 \mathrm{M}_{\odot}$, radii, $R_{1}=1.70 \pm 0.03 \mathrm{R}_{\odot}$ and $R_{2}=1.38 \pm 0.03 \mathrm{R}_{\odot}$, and effective temperatures, $T_{1}=6650 \pm 200 \mathrm{~K}$ and $T_{2}=6235 \pm 201 \mathrm{~K}$. Considering interstellar extinction the distance to V355 And was computed as $117 \pm 7 \mathrm{pc}$. The evolutionary state of V355 And is also discussed using the Geneva stellar models.
\end{abstract}

Key words: Stars: binaries: eclipsing; Stars: absolute parameters; Stars: Individual V355 And

\section{Introduction}

Eclipsing binary systems are important celestial objects in astrophysics in determining the astrophysical parameters of stars in our galaxy, the Milky Way, and nearby galaxies and understanding stellar evolutionary status. Since the proximity and ellipsoidal effects are not important in detached binary systems, their absolute parameters can be derived more accurately from the solutions of light and radial velocity curves. This paper focuses on the neglected eclipsing binary V355 And. The primary objectives of the present study are to determine the absolute dimensions of V355 And and to examine the evolutionary state of the system's components. Literature-based studies of this neglected binary star are summarized below.

V355 And is an Algol-type binary star and a component of the visual binary WDS 00442-4614. The eclipsing nature and multiplicity of the system were 
discovered by the Hipparcos satellite (ESA, 1997). The system has a fainter $\left(\Delta \mathrm{V}_{H i p} \simeq 1^{m} .17\right)$ component at a separation of 1.41 arcsec and a position angle of 5.5 deg. The target system V355 And is one of the poorly studied eclipsing binary stars. Kazarovets et al. (1999) gave the GCVS name and variability type of the system in their 74th name-list of variable stars. Tikkanen (2002) published the complete visual light curve of V355 And and revealed the light elements to be $T_{0}=2452295.088(8)$ and $P=4.71841(2)$. Imbert (2006) determined the spectroscopic orbits of 25 binary stars, including the double-lined system V355 And, using 1402 Coravel radial velocity measurements. Zasche et al. (2009) presented a new catalog of visual double systems containing eclipsing binaries as one component. For V355 And, they suggested the visual orbit's period to be in the order of $3000 \mathrm{yr}$ according to the orbital coverage. Hubscher (2015) recorded one minimum light time in the $I$-filter for the system. Some photometric properties of the multiple system V355 And are given in various catalogues (e.g. Malkov et al., 2006; McDonald et al., 2012; Avvakumova et al., 2013; Paunzen, 2015; Soubiran et al., 2016). The fundamental data for V355 And is given in Table 1.

Table 1. Fundamental data based on the ASAS-SN database for V355 And.

\begin{tabular}{ll}
\hline \hline Star Name & ASASSN- $V$ J004411.33+461407.2 \\
\hline LCID & 51037 \\
RA, DEC & $11.0472,46.2353$ \\
Epoch (HJD) & 2457747.80809 \\
$V$ mag & 7.97 \\
Amplitude & 0.73 \\
Type & VAR \\
\hline
\end{tabular}

\section{O-C analysis}

\subsection{Data}

For V355 And, there are few times of minimum light in the literature: Tikkanen (2002) observed the system visually and obtained 5 minima times. In the $O-C$ Gateway archive ${ }^{1}, 4$ minima times of the eclipsing binary V355 And are recorded. In this study, 9 minima times of the system were obtained from the photometric light curves of the SuperWASP (Wide Angle Search for Planets; Butters et al., 2010) and TESS (Transiting Exoplanet Survey Satellite; Ricker et al., 2015) surveys. Newly determined minima times were computed with the software Minima Vers.27 published by B. Nelson ${ }^{2}$ using the Kwee \& van Woer-

\footnotetext{
${ }^{1}$ http://var2.astro.cz/ocgate/index.php?lang=en

${ }^{2}$ https://www.variablestarssouth.org/software-by-bob-nelson/
} 
den (1956) method. All minima times in HJD were converted to BJD ${ }_{\mathrm{TDB}}$ using the HJD2BJD calculator ${ }^{3}$ (Eastman et al., 2010) because the calculated times of minima from TESS observations were in the BJD $_{\text {TDB }}$ time system. Minima times of V355 And are tabulated in Table 2.

Table 2. Minima times of V355 And.

\begin{tabular}{lccc}
\hline \hline BJD $_{\text {тDв }}(+2400000)$ & Type & Method & Reference \\
\hline $52271.5000 \pm 0.0210$ & $\mathrm{p}$ & visual & \\
$52276.2170 \pm 0.0160$ & $\mathrm{p}$ & visual & \\
$52283.2910 \pm 0.0070$ & $\mathrm{p}$ & visual & Tikkanen $(2002)$ \\
$52295.0900 \pm 0.0080$ & $\mathrm{p}$ & visual & \\
$52309.2420 \pm 0.0110$ & $\mathrm{~s}$ & visual & \\
\hline $56928.5218 \pm 0.0033$ & $\mathrm{p}$ & $\mathrm{CCD}$ & \\
$57256.4517 \pm 0.0064$ & $\mathrm{~s}$ & $\mathrm{CCD}$ & $\mathrm{O}-\mathrm{C}$ Gateway \\
$57992.5188 \pm 0.0015$ & $\mathrm{~s}$ & $\mathrm{CCD}$ & \\
$58379.4208 \pm 0.0015$ & $\mathrm{~s}$ & $\mathrm{CCD}$ & \\
\hline $54418.3577 \pm 0.0004$ & $\mathrm{p}$ & $\mathrm{CCD}$ & SuperWASP \\
$54373.5245 \pm 0.0004$ & $\mathrm{~s}$ & $\mathrm{CCD}$ & \\
\hline $58768.6916 \pm 0.0001$ & $\mathrm{p}$ & $\mathrm{CCD}$ & \\
$58778.1289 \pm 0.0002$ & $\mathrm{p}$ & $\mathrm{CCD}$ & \\
$58782.8474 \pm 0.0001$ & $\mathrm{p}$ & $\mathrm{CCD}$ & \\
$58766.3361 \pm 0.0002$ & $\mathrm{~s}$ & $\mathrm{CCD}$ & TESS \\
$58771.0540 \pm 0.0001$ & $\mathrm{~s}$ & $\mathrm{CCD}$ & \\
$58780.4909 \pm 0.0001$ & $\mathrm{~s}$ & $\mathrm{CCD}$ & \\
$58785.2092 \pm 0.0001$ & $\mathrm{~s}$ & $\mathrm{CCD}$ & \\
\hline \hline
\end{tabular}

\subsection{New light elements}

We can examine whether the orbital period of a binary system has changed using the $O-C$ diagram. In the case of V355 And, the $O-C$ values of the minima times listed in Table 2 were calculated using $T_{0}=2458768.6916$ (from TESS observations) and $P=4.71841$ (from Tikkanen, 2002), and thus the $O$ - $C$ diagram of the system was created. A linear regression of all $O-C$ values was performed to obtain the revised ephemeris, given in equation (1). The residuals are plotted in Fig. 1. In this figure, it is clearly seen that the period of the system has been constant throughout the time interval of 18 years.

$\operatorname{Min} I\left(B J D_{\mathrm{TDB}}\right)=2458768.6924( \pm 0.0011)+4^{d} .7183707( \pm 0.0000013) \times E$,

where the values in brackets are the uncertainties.

${ }^{3} \mathrm{http}: / /$ astroutils.astronomy.ohio-state.edu/time/hjd2bjd.html 


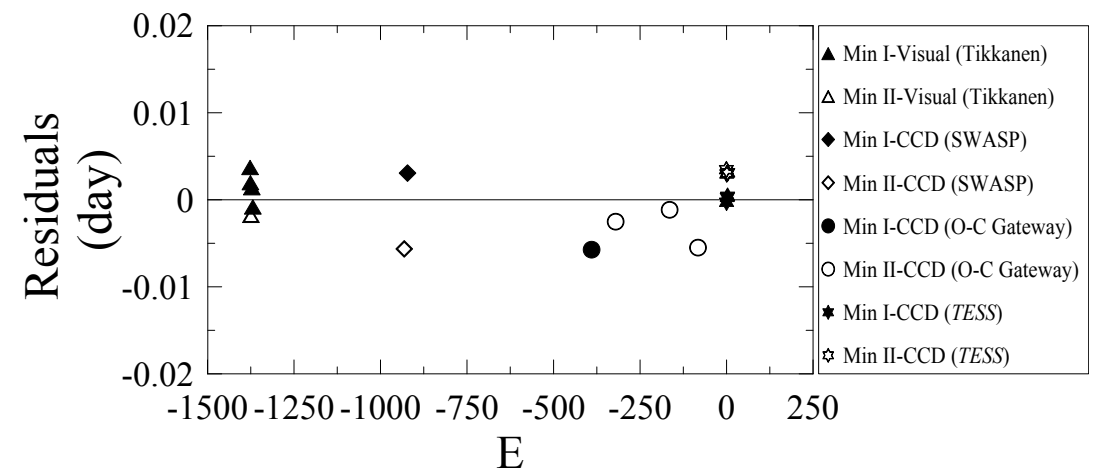

Figure 1. A plot of $O-C$ residuals versus the ephemeris used in equation (1).

\section{Light curve modeling}

\subsection{Data}

The neglected binary V355 And was observed by five different photometric surveys: The All-Sky Automated Survey for Supernovae (ASAS-SN; Shappee et al., 2014; Kochanek et al., 2017; Jayasinghe et al., 2018), Kamogata/Kiso/Kyoto Wide-field Survey (KWS; Maehara, 2014), Hipparcos, SuperWASP and TESS. The photometric light curves observed by the TESS ${ }^{4}, \mathrm{ASAS}_{-} \mathrm{SN}^{5}$ and $\mathrm{KWS}^{6}$ were analyzed, while the Hipparcos and SuperWASP light curves were not solved because of the inadequate phase covering and more scattered data.

The main purpose of TESS is to explore planetary transits by scanning the chosen sky over a period of two years. TESS observational data comes from sectors that cover a $24^{\circ} \times 90^{\circ}$ region in the sky. The observations of 32 sectors in total have been completed so far. TESS observations of V355 And $\left(\mathrm{T}_{\text {mag }}=7.42\right.$, TIC 601366244) were made in $120 \mathrm{~s}$ cadence during Sector 17 starting in October 2019. In this study, Simple Aperture Photometry (SAP) Flux data with QUALITY $=0$ of V355 And, which consists of 12,613 photometric points for the system, was converted into magnitude values. The average values per each three data points were used to generate the normalized data, thus nearly 4200 normal points in total. The photometric phases of the TESS light curve were computed using equation (1).

The ASAS-SN project aims to survey the whole sky up to 18th magnitude and focuses on the discovery of bright nearby supernovae. In the ASAS-SN Variable Stars database 666,502 light curves are available, including the $V$ band light curve of the target binary V355 And obtained between October

\footnotetext{
${ }^{4}$ https://mast.stsci.edu/portal/Mashup/Clients/Mast/Portal.html

${ }^{5}$ https://asas-sn.osu.edu/

${ }^{6}$ http://kws.cetus-net.org/ maehara/VSdata.py
} 
2013 and January 2018. The ASAS-SN light curve of V355 And is composed of about 1800 data points in $V$ mag. The outliers with $\sigma \geq 0.0004$, where $\sigma=\frac{\text { error of observation point }}{\text { observation point }}$, were removed from the ASAS-SN data. The remaining data points, $\sim 1480$, were processed in the analysis. Using equation (1), the phases of the ASAS-SN light curve were calculated.

KWS is designed for the automated wide-field optical survey of bright transits and variable stars via a small CCD camera and lens to achieve a photometric precision of $<5 \%$ for stars with $V$-band magnitude between 5 and 11 . KWS observations of V355 And were made between December 2010 and January 2020, and nearly 1200 data points were obtained in total in the $V$ and $I_{c}$ filters. The scattered data points with $\sigma \geq 0.00095$ for $V$ and $\sigma \geq 0.00013$ for $I_{c}$ were removed from the KWS data, thus a total of $\sim 800$ data points remained for the analysis. Equation (1) was used for phasing the KWS- $V I_{c}$ light curves.

The spectroscopic orbital parameters of the system given by Imbert (2006) are tabulated in Table 3 . The spectroscopic mass ratio $\left(q=\frac{K_{1}}{K_{2}}\right)$ of V355 And is calculated to be 0.88 based on this table. Public data of the radial velocity curves of the eclipsing pair can be extracted from the webpage ${ }^{7}$ of SB9 (Pourbaix et al., 2004).

Table 3. Spectroscopic orbital parameters of V355 And from Imbert (2006).

\begin{tabular}{llcc}
\hline \hline Parameters & Unit & Primary & Secondary \\
\hline T0 & HJD & & $48929.67 \pm 0.007$ \\
Period & day & & $4.71835 \pm 0.00002$ \\
$\mathrm{e}$ & - & & 0 (fixed) \\
$\omega$ & $\mathrm{deg}$ & & 0 (fixed) \\
$\mathrm{K}$ & $\mathrm{km} / \mathrm{s}$ & $85.01 \pm 0.28$ & $96.59 \pm 0.30$ \\
$\mathrm{~V}_{\gamma}$ & $\mathrm{km} / \mathrm{s}$ & \multicolumn{2}{c}{$6.93 \pm 0.17$} \\
$\operatorname{asin}(\mathrm{i})$ & $\mathrm{km}$ & $5.52 \times 10^{6} \pm 0.02 \times 10^{6}$ & $6.27 \times 10^{6} \pm 0.02 \times 10^{6}$ \\
$\min ^{3}(\mathrm{i})$ & $\mathrm{M}_{\odot}$ & $1.56 \pm 0.01$ & $1.38 \pm 0.01$ \\
\hline \hline
\end{tabular}

\subsection{Analysis}

If both a light curve and radial velocity curves of an eclipsing binary system are obtained, the absolute parameters of that system can be determined more precisely. The Wilson-Devinney (WD) code (Wilson \& Devinney, 1971), appended with the Monte Carlo (MC) algorithm (Zola et al., 2010), was used for the light curve modeling. In the first step, due to its high inherent accuracy (each datum on the order of $0.0001 \mathrm{mag}$ ), the TESS light curve of V355 And was solved. The MC procedure outlined by Zola et al. (2010) was followed, and the spectroscopic mass ratio value was kept fixed. In the second step, ASAS-SN and

${ }^{7}$ https://sb9.astro.ulb.ac.be/ 
KWS light curves were solved. The ASAS-SN and KWS light curves both have lower photometric accuracy and show more scattering at their maximum levels (see Fig. 2). On the other hand, there are not enough observation points in the depths of the minima of KWS light curves. As it is well known, the depths of minima of a given light curve are important factors in determining the surface potential parameters (then the fractional radii) of component stars. Therefore, in the solution of ASAS-SN and KWS light curves, these surface potential parameters $\left(\Omega_{1}, \Omega_{2}\right)$ were fixed at the values obtained from the solution of the TESS light curve, and the other parameters were adjusted.

The following parameters were adopted and fixed during all iterations. The temperature of the primary component was fixed at $6650 \mathrm{~K}$ corresponding to the F5 spectral type given by the Hipparcos catalogue using Drilling \& Landolt (2000)'s calibration for MK spectral types. The bolometric gravity-darkening exponents $\left(g_{1}=g_{2}=0.32\right)$ and albedos $\left(A_{1}=A_{2}=0.5\right)$ of the components were set at their theoretical values as suitable for the spectral type of component stars, following Lucy (1967) and Ruciński (1969), respectively. The square-root limbdarkening law was adopted with limb-darkening coefficients from the calibration tables of Claret \& Bloemen (2011) and Claret et al. (2013), depending on the filter wavelengths and temperatures of the primary and secondary components. Besides synchronous rotation of the components, a circular orbit for the systems was also assumed.

The free parameters in the light curve fitting were the orbital inclination $(i)$, phase-shift $(\phi)$, temperature of the secondary component $\left(T_{2}\right)$, non-dimensional surface potential parameters of components $\left(\Omega_{1}\right.$ and $\left.\Omega_{2}\right)$, luminosity of the primary $\left(L_{1}\right)$ and third light contribution $\left(\frac{L_{3}}{L_{\text {total }}}\right)$. In the MC process, an assumed range within realistic bounds for each free parameter should be defined. In the analysis, the search ranges of the adjusted parameters are given in Table 4.

The parameters of the WD+MC solution are listed in Table 4 with the errors corresponding to a $90 \%$ confidence level. The best theoretical fits of the $\mathrm{WD}+\mathrm{MC}$ modeling program are compared with the observed light curves in Fig. 2. The 2D plot of the parameter space for degeneracies between the chosen five parameters $\left(i, T_{2}, \Omega_{1}, \Omega_{2}\right.$ and $\left.L_{3}\right)$ are shown in Fig. 3 using MC confidence levels. There are considerable degeneracies between $\Omega_{1}$ and $\Omega_{2}$ and between $i$ and $L_{3}$, while no degeneracies are seen between $i$ and $T_{2}, i$ and $\Omega_{1}$ and $i$ and $\Omega_{2}$. Such degeneracies between the geometric and physical parameters were found in some detached binaries (see Garcia et al., 2014; Sürgit et al., 2017).

Table 4. Selected search ranges of adjusted parameters in the WD+MC method.

\begin{tabular}{lllllll}
\hline \hline System & $i(\mathrm{deg})$ & $\phi$ & $T_{2}(\mathrm{~K})$ & $\Omega_{1}$ and $\Omega_{1}$ & $L_{1}$ & $\frac{L_{3}}{L_{\text {total }}}$ \\
\hline V355 And & $70-90$ & $-0.01-0.01$ & $5500-6600$ & $9.0-14.0$ & $2.0-12.0$ & $0.1-0.50$ \\
\hline \hline
\end{tabular}


Table 5. Results of the analysis of light curves of V355 And.

\begin{tabular}{llll}
\hline \hline Parameters & TESS & ASAS-SN & KWS \\
\hline$i\left(^{\circ}\right)$ & $88.1 \pm 0.2$ & $87.5 \pm 0.2$ & $88.1 \pm 0.2$ \\
$\phi$ & $-0.0006 \pm 0.0001$ & $-0.0007 \pm 0.0001$ & $-0.0007 \pm 0.0001$ \\
$T_{1}(K)$ & 6650 (fixed) & 6650 & 6650 \\
$T_{2}(K)$ & $6414 \pm 12$ & $6237 \pm 14$ & $6297 \pm 23$ \\
$q=M_{2} / M_{1}$ & 0.88 (fixed) & 0.88 & 0.88 \\
$\Omega_{1}$ & $10.867 \pm 0.109$ & 10.867 & 10.867 \\
$\Omega_{2}$ & $11.838 \pm 0.191$ & 11.838 & 11.838 \\
$r_{1}($ mean $)$ & $0.100 \pm 0.001$ & 0.100 & 0.100 \\
$r_{2}($ mean $)$ & $0.082 \pm 0.001$ & 0.082 & 0.082 \\
$L_{1} / L_{\text {total }}-T E S S$ & $0.45 \pm 0.02$ & - & - \\
$L_{1} / L_{\text {total }}-V$ & - & $0.46 \pm 0.01$ & $0.45 \pm 0.02$ \\
$L_{1} / L_{\text {total }}-I_{c}$ & - & - & $0.46 \pm 0.01$ \\
$L_{3} / L_{\text {total }}-T E S S$ & $0.29 \pm 0.02$ & - & - \\
$L_{3} / L_{\text {total }}-V$ & - & $0.30 \pm 0.01$ & $0.28 \pm 0.01$ \\
$L_{3} / L_{\text {total }}-I_{c}$ & - & - & $0.28 \pm 0.01$ \\
$\sum W(O-C)^{2}-R V 1$ & 0.0003 & 0.0003 & 0.0003 \\
$\sum W(O-C)^{2}-R V 2$ & 0.0003 & 0.0003 & 0.0003 \\
$\sum W(O-C)^{2}-L C-T E S S$ & 0.0194 & - & - \\
$\sum \sum W(O-C)^{2}-L C-V$ & - & 0.2548 & 0.2843 \\
$\sum W(O-C)^{2}-L C-I_{c}$ & - & - & 0.1731 \\
\hline \hline
\end{tabular}

\section{Results and discussion}

A detailed photometric data analysis of V355 And was performed in this study for the first time. The best-fitting model reveals that V355 And has a detached configuration, and the primary and secondary components fill $34 \%$ and $31 \%$ of their Roche limiting lobes, respectively. In the following subsections, the obtained absolute parameters and evolutionary status of the system are given.

\subsection{Absolute dimensions}

The absolute dimensions of V355 And were calculated from the combination of the TESS light curve solution and the RV curve solution, and are given in Table 6 with their uncertainties. The solar values, $T_{\text {eff }}=5771.8 \pm 0.7 \mathrm{~K}$, $M_{b o l}=4.7554 \pm 0.0004 \mathrm{mag}$ and $g=27423.2 \pm 7.9 \mathrm{~cm} / \mathrm{s}^{2}$, taken from Pecaut \& Mamajek (2013), were used in the computations.

The uncertainty of $20 \mathrm{~K}$ in the temperature of the secondary component, seen in Table 5, corresponds to the 1- $\sigma$ formal error resulting from the WD+MC analysis. The standard error of the secondary component's temperature has been corrected to $201 \mathrm{~K}$ by adopting the standard error of $200 \mathrm{~K}$ in the temperature of the primary component given in Table 6. The Bolometric corrections (BC) 


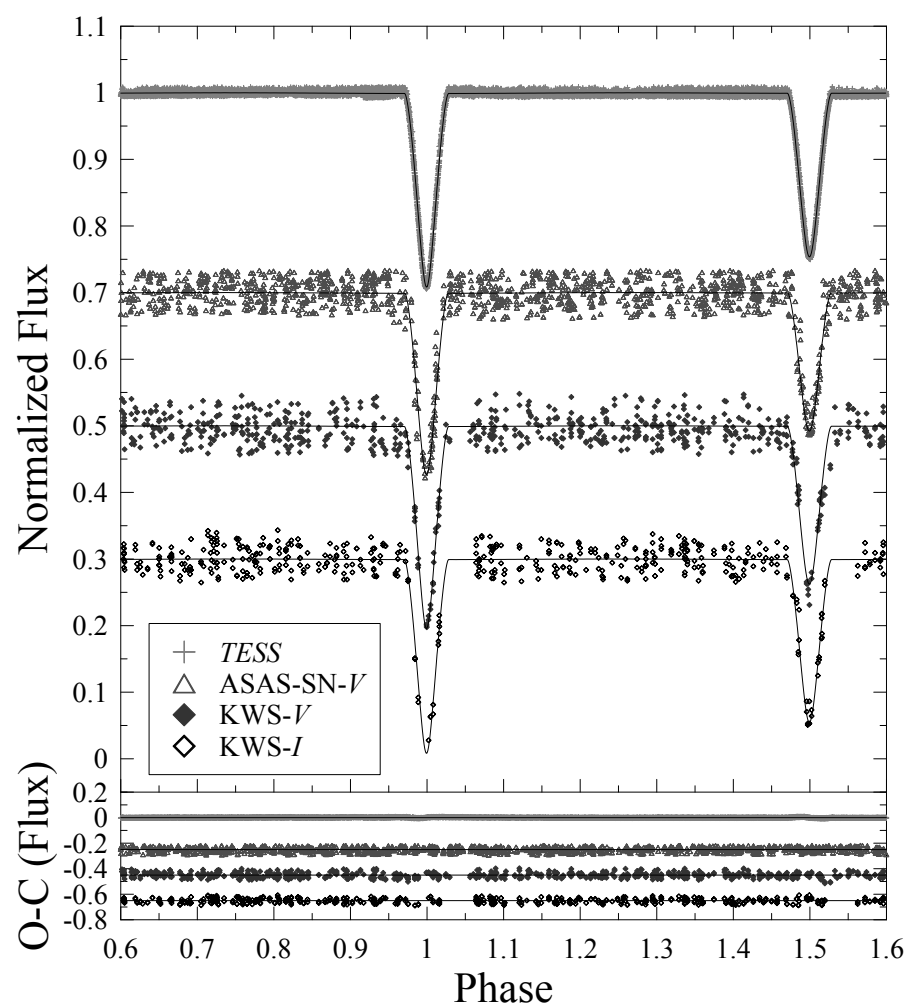

Figure 2. Comparison between the theoretical (solid lines) and TESS (top), ASAS-SN- $V$ (middle) and KWS- $V I_{c}$ light curves (bottom) of V355 And. The residuals of the WD+MC model fitting are shown in the bottom panel.

of the primary and secondary components were chosen from the tabulation of Flower (1996) to be $B C_{1}=0.016$ and $B C_{2}=-0.017$, respectively, according to the computed effective temperatures of the components. The absolute magnitudes of both components were then obtained.

Through the well-known equation $E(B-V)=(B-V)-(B-V)_{0}$, the color excess of the system was determined as 0.017 , where the de-reddened color index $(\mathrm{B}-\mathrm{V})_{0}$ was computed using the absolute magnitude calibrations for detached binaries given by Bilir et al. (2008), while the $(B-V)$ value of the system was taken from the APASS catalogue ${ }^{8}$. The interstellar extinction value $A_{\nu}$ in the $V$ passband was computed as 0.052 mag by means of the well-known formula $A_{V}=3.1 \times E(B-V)$. Finally, the distance to V355 And of $117 \pm 7$ pc was derived from the distance modulus formula. Additionally, the distance of the

${ }^{8}$ APASS Catalogue: The AAVSO Photometric All-Sky Survey, http://www.aavso.org/apass. 

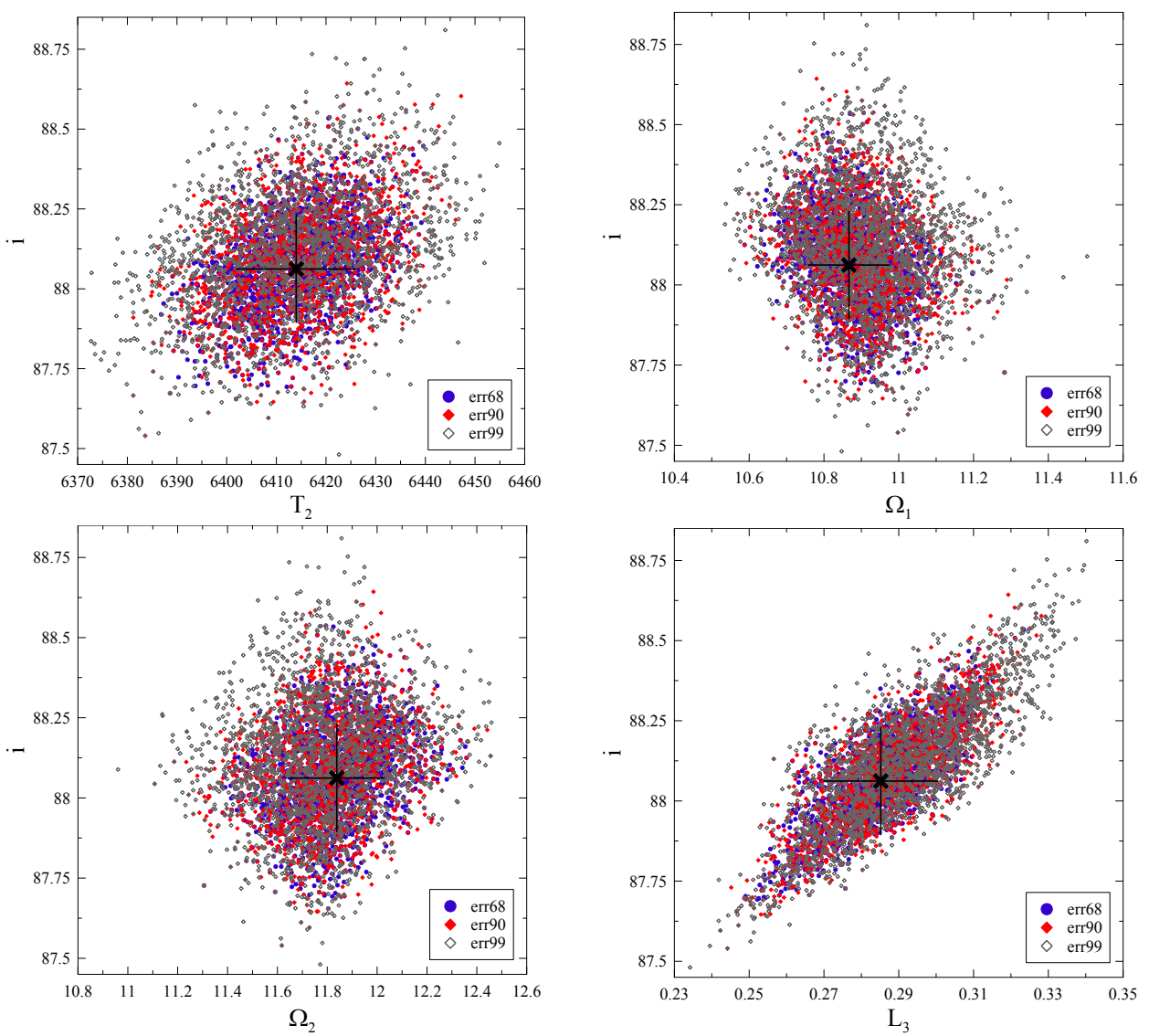

$\Omega_{2}$

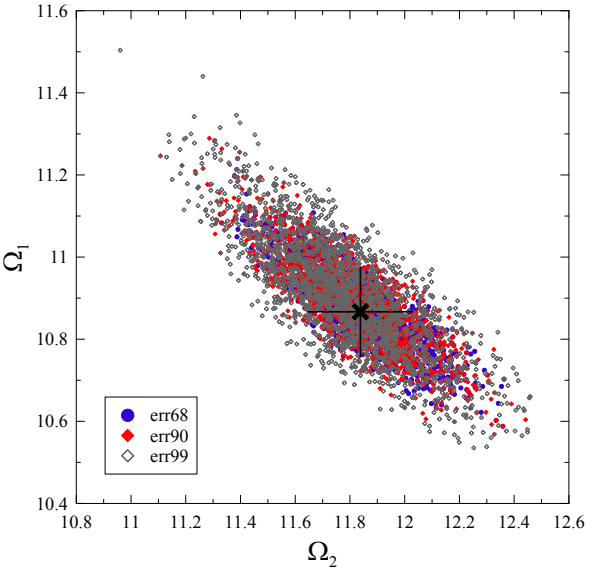

Figure 3. Degeneracies of the selected parameters, $\left(i, T_{2}, \Omega_{1}, \Omega_{2}\right.$ and $\left.L_{3}\right)$, for the $\mathrm{WD}+\mathrm{MC}$ search best fit solution using three confidence levels $(68 \%, 90 \%$, and $99 \%)$. Confidence levels are represented by blue-filled circles, red diamonds and grey diamonds. The best-fit solution is represented by the cross symbol with error bars selected from a $90 \%$ confidence level. 
system was calculated to be $117.9 \pm 2.0 \mathrm{pc}$ according to the Gaia DR2 parallax (Gaia Collaboration et al., 2018). It is clearly seen that the determined distance is in good agreement when compared with the Gaia distance.

Table 6. Absolute parameters of the components of V355 And.

\begin{tabular}{llcc}
\hline \hline Parameters & Unit & Primary & Secondary \\
\hline$A$ & $\mathrm{R}_{\odot}$ & \multicolumn{2}{c}{$16.94 \pm 0.03$} \\
$P_{\text {orb }}$ & days & $4.7183808 \pm 0.0000001)$ \\
$M$ & $\mathrm{M}_{\odot}$ & $1.56 \pm 0.01$ & $1.38 \pm 0.01$ \\
$R$ & $\mathrm{R}_{\odot}$ & $1.70 \pm 0.03$ & $1.38 \pm 0.03$ \\
$\log g$ & cgs & $4.17 \pm 0.02$ & $4.29 \pm 0.01$ \\
$T$ & $\mathrm{~K}$ & $6650 \pm 200$ & $6235 \pm 201$ \\
$M_{\text {bol }}$ & mag & $2.99 \pm 0.16$ & $3.59 \pm 0.14$ \\
$M_{V}$ & mag & $2.97 \pm 0.16$ & $3.61 \pm 0.14$ \\
$L$ & $\mathrm{~L} \odot$ & $5.10 \pm 0.77$ & $2.92 \pm 0.37$ \\
$M_{V}$ & mag & \multicolumn{2}{c}{$2.10 \pm 0.13$} \\
$B C$ & $\mathrm{mag}$ & 0.016 & -0.017 \\
$d$ & $\mathrm{pc}$ & \multicolumn{2}{c}{$117 \pm 7$} \\
\hline \hline
\end{tabular}

\subsection{Evolutionary status}

A comparison of the measured physical parameters of a star with predictions from theoretical models can be made by means of the Hertzsprung-Russell (HR) diagram. According to the iron number abundance, $[\mathrm{Fe} / \mathrm{H}]=0.17$, given by Nordström et al. (2004) and Holmberg et al. (2009), the metallicity value was computed to be 0.021 using the relation between $\mathrm{Z}$ and $[\mathrm{Fe} / \mathrm{H}]$ in the logarithmic scale $^{9}$. Therefore, the locations of the components of V355 And were plotted on the $\log T_{\text {eff }}-\log \left(L / L_{\odot}\right)$ diagram (see Fig. 4$)$, namely the HR diagram, using the Geneva stellar evolution models ${ }^{10}$ (Mowlavi et al., 2012) for the metallicity $\mathrm{Z}=0.02$. Trials were made for other $\mathrm{Z}$ values $(\mathrm{Z}=0.14$ and $\mathrm{Z}=0.03)$; however, as it can be seen in Fig. 4, the theoretical evolution model at $Z=0.02$ is the most suitable for the masses of both components of the system. In addition, we also tried to predict the age of V355 And using the evolutionary isochrones for $\mathrm{Z}=0.02$ adopted from Mowlavi et al. (2012). Consequently, with respect to the HR diagram, it can be said that the components of V355 And are main-sequence stars at the age of $\sim 1.26$ Gyr.

\footnotetext{
${ }^{9}$ https://people.umass.edu/wqd/astro640/ToO.pdf

${ }^{10}$ https://www.unige.ch/sciences/astro/evolution/en/database/syclist/
} 


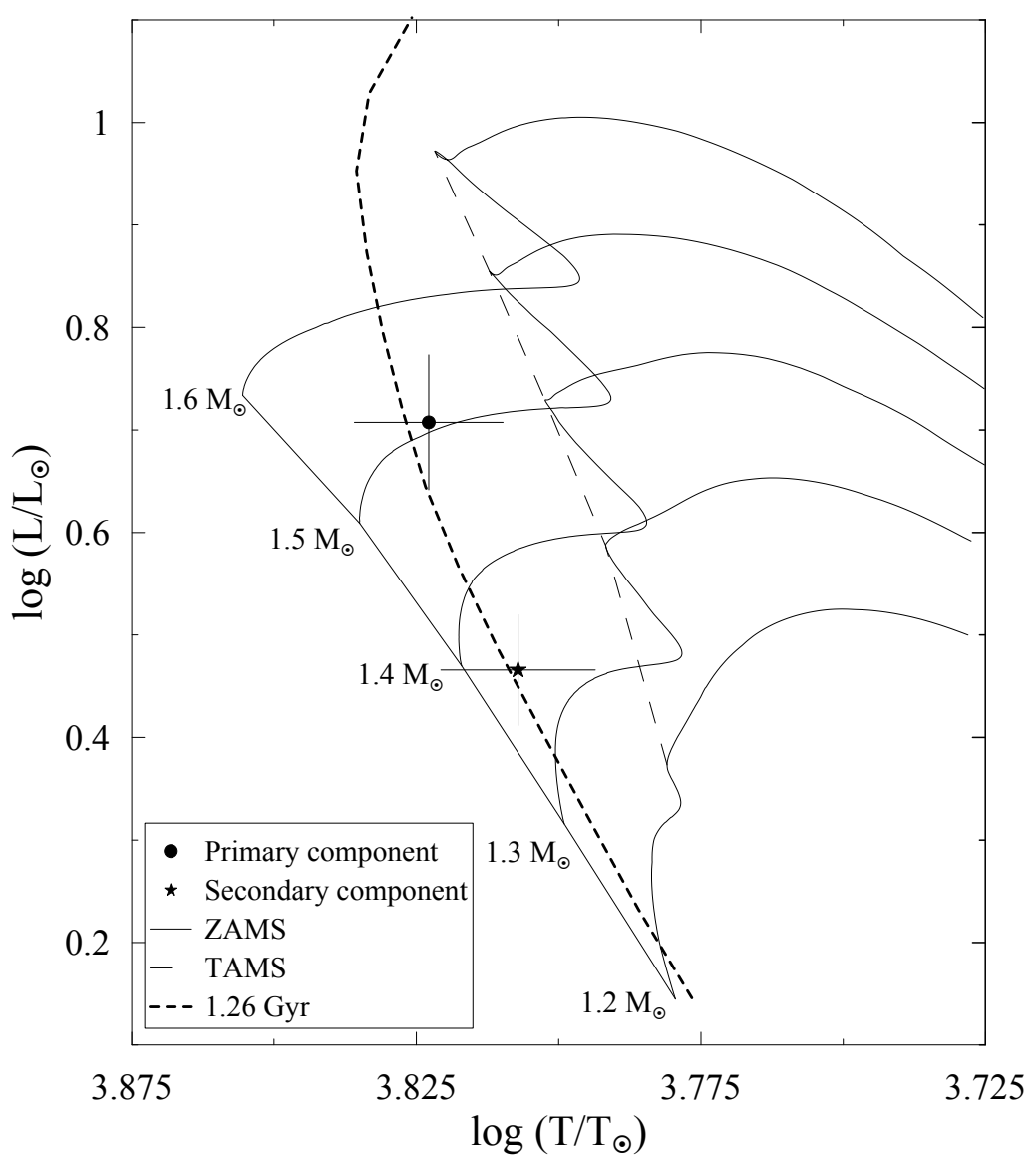

Figure 4. Locations of primary and secondary components of V355 And in the logarithmic temperature-luminosity plane according to Geneva stellar evolutionary models. ZAMS, TAMS and evolutionary tracks were taken from Mowlavi et al. (2012) for the metallicity of $\mathrm{Z}=0.02$. Lines drawn vertically and horizontally denote error bars of the measured parameters. The isochrone for the estimated age of the system at $1.26 \mathrm{Gyr}$ is represented by a dashed line. In the HR diagram the numbers added to the left of ZAMS indicate initial masses.

Acknowledgements. This paper includes data collected by the TESS mission, which are publicly available from the Mikulski Archive for Space Telescopes (MAST). The author would like to thank Prof. A. Erdem for his advice and comments, and Prof. $\mathrm{S}$. Zola for his help in using the W-D FORTRAN code with modern limb-darkening coefficients. The author would like to thank the anonymous referee who provided valuable comments for improving the manuscript. The author also thanks Mr. G.H. Lee for checking the English. 


\section{References}

Avvakumova, E. A., Malkov, O. Y., \& Kniazev, A. Y., Eclipsing variables: Catalogue and classification. 2013, Astronomische Nachrichten, 334, 860, DOI: 10.1002/asna.201311942

Bilir, S., Ak, T., Soydugan, E., et al., New absolute magnitude calibrations for detached binaries. 2008, Astronomische Nachrichten, 329, 835, DOI: 10.1002/asna.200811002

Butters, O. W., West, R. G., Anderson, D. R., et al., The first WASP public data release. 2010, Astronomy and Astrophysics, 520, L10, DOI: 10.1051/0004$6361 / 201015655$

Claret, A. \& Bloemen, S., Gravity and limb-darkening coefficients for the Kepler, CoRoT, Spitzer, uvby, UBVRIJHK, and Sloan photometric systems. 2011, Astronomy and Astrophysics, 529, A75, DOI: 10.1051/0004-6361/201116451

Claret, A., Hauschildt, P. H., \& Witte, S., New limb-darkening coefficients for Phoenix/1d model atmospheres. II. Calculations for $5000 \mathrm{~K} \leq \mathrm{T}_{\text {eff }} \leq 10000 \mathrm{~K} \mathrm{Ke}-$ pler, CoRot, Spitzer, uvby, UBVRIJHK, Sloan, and 2MASS photometric systems. 2013, Astronomy and Astrophysics, 552, A16, DOI: 10.1051/0004-6361/201220942

Drilling, J. S. \& Landolt, A. U., Normal Stars. 2000, in Allen's astrophysical quantities, ed. A. N. Cox (Springer-Verlag New York), 381-396

Eastman, J., Siverd, R., \& Gaudi, B. S., Achieving Better Than 1 Minute Accuracy in the Heliocentric and Barycentric Julian Dates. 2010, Publications of the ASP, 122, 935, DOI: $10.1086 / 655938$

ESA, VizieR Online Data Catalog: The Hipparcos and Tycho Catalogues (ESA 1997). 1997, VizieR Online Data Catalog, I/239

Flower, P. J., Transformations from Theoretical Hertzsprung-Russell Diagrams to Color-Magnitude Diagrams: Effective Temperatures, B-V Colors, and Bolometric Corrections. 1996, Astrophysical Journal, 469, 355, DOI: 10.1086/177785

Gaia Collaboration, Brown, A. G. A., Vallenari, A., et al., Gaia Data Release 2. Summary of the contents and survey properties. 2018, Astronomy and Astrophysics, 616, A1, DOI: $10.1051 / 0004-6361 / 201833051$

Garcia, E. V., Stassun, K. G., Pavlovski, K., et al., A Strict Test of Stellar Evolution Models: The Absolute Dimensions of the Massive Benchmark Eclipsing Binary V578 Mon. 2014, Astronomical Journal, 148, 39, DOI: 10.1088/0004-6256/148/3/39

Holmberg, J., Nordström, B., \& Andersen, J., The Geneva-Copenhagen survey of the solar neighbourhood. III. Improved distances, ages, and kinematics. 2009, Astronomy and Astrophysics, 501, 941, DOI: 10.1051/0004-6361/200811191

Hubscher, J., BAV-Results of observations - Photoelectric Minima of Selected Eclipsing Binaries and Maxima of Pulsating Stars. 2015, Information Bulletin on Variable Stars, 6152, 1

Imbert, M., Vitesses Radiales, Elements Orbitaux et Modeles Pour 25 Systemes Doubles Spectroscopiques. 2006, Romanian Astronomical Journal, 16, 3 
Jayasinghe, T., Kochanek, C. S., Stanek, K. Z., et al., The ASAS-SN catalogue of variable stars I: The Serendipitous Survey. 2018, Monthly Notices of the RAS, 477, 3145, DOI: $10.1093 /$ mnras/sty838

Kazarovets, E. V., Samus, N. N., Durlevich, O. V., et al., The 74th Special Name-list of Variable Stars. 1999, Information Bulletin on Variable Stars, 4659, 1

Kochanek, C. S., Shappee, B. J., Stanek, K. Z., et al., The All-Sky Automated Survey for Supernovae (ASAS-SN) Light Curve Server v1.0. 2017, Publications of the ASP, 129, 104502, DOI: 10.1088/1538-3873/aa80d9

Kwee, K. K. \& van Woerden, H., A method for computing accurately the epoch of minimum of an eclipsing variable. 1956, Bulletin Astronomical Institute of the Netherlands, 12, 327

Lucy, L. B., Gravity-Darkening for Stars with Convective Envelopes. 1967, Zeitschrift fuer Astrophysik, 65, 89

Maehara, H., Automated Wide-field Survey for Transient Objects with a Small Telescope. 2014, JAXA research and development report, JAXA-RR-13-010, 119

Malkov, O. Y., Oblak, E., Snegireva, E. A., \& Torra, J., A catalogue of eclipsing variables. 2006, Astronomy and Astrophysics, 446, 785, DOI: 10.1051/00046361:20053137

McDonald, I., Zijlstra, A. A., \& Boyer, M. L., Fundamental parameters and infrared excesses of Hipparcos stars. 2012, Monthly Notices of the RAS, 427, 343, DOI: 10.1111/j.1365-2966.2012.21873.x

Mowlavi, N., Eggenberger, P., Meynet, G., et al., Stellar mass and age determinations . I. Grids of stellar models from $\mathrm{Z}=0.006$ to 0.04 and $\mathrm{M}=0.5$ to $3.5 \mathrm{M}$. 2012, Astronomy and Astrophysics, 541, A41, DOI: 10.1051/0004-6361/201117749

Nordström, B., Mayor, M., Andersen, J., et al., The Geneva-Copenhagen survey of the Solar neighbourhood. Ages, metallicities, and kinematic properties of $\sim 14000$ F and G dwarfs. 2004, Astronomy and Astrophysics, 418, 989, DOI: 10.1051/00046361:20035959

Paunzen, E., A new catalogue of Strömgren-Crawford uvby $\beta$ photometry. 2015, Astronomy and Astrophysics, 580, A23, DOI: 10.1051/0004-6361/201526413

Pecaut, M. J. \& Mamajek, E. E., Intrinsic Colors, Temperatures, and Bolometric Corrections of Pre-main-sequence Stars. 2013, Astrophysical Journal, Supplement, 208, 9, DOI: $10.1088 / 0067-0049 / 208 / 1 / 9$

Pourbaix, D., Tokovinin, A. A., Batten, A. H., et al., $\mathrm{S}_{B^{9}}$ : The ninth catalogue of spectroscopic binary orbits. 2004, Astronomy and Astrophysics, 424, 727, DOI: 10.1051/0004-6361:20041213

Ricker, G. R., Winn, J. N., Vanderspek, R., et al., Transiting Exoplanet Survey Satellite (TESS). 2015, Journal of Astronomical Telescopes, Instruments, and Systems, 1, 014003, DOI: 10.1117/1.JATIS.1.1.014003

Ruciński, S. M., The Proximity Effects in Close Binary Systems. II. The Bolometric Reflection Effect for Stars with Deep Convective Envelopes. 1969, Acta Astronomica, 19, 245 
Shappee, B. J., Prieto, J. L., Grupe, D., et al., The Man behind the Curtain: XRays Drive the UV through NIR Variability in the 2013 Active Galactic Nucleus Outburst in NGC 2617. 2014, Astrophysical Journal, 788, 48, DOI: 10.1088/0004$637 \mathrm{X} / 788 / 1 / 48$

Soubiran, C., Le Campion, J.-F., Brouillet, N., \& Chemin, L., The PASTEL catalogue: 2016 version. 2016, Astronomy and Astrophysics, 591, A118, DOI: 10.1051/0004$6361 / 201628497$

Sürgit, D., Erdem, A., Engelbrecht, C. A., van Heerden, H. P., \& Manick, R., Absolute parameters of detached binaries in the southern sky - III: HO Tel. 2017, New Astronomy, 54, 109, DOI: 10.1016/j.newast.2017.01.013

Tikkanen, A., 160. list of minima of eclipsing binaries. 2002, Bulletin der Bedeckungsveraenderlichen-Beobachter der Schweizerischen Astronomischen Gesellschaft, 127, 1

Wilson, R. E. \& Devinney, E. J., Realization of Accurate Close-Binary Light Curves: Application to MR Cygni. 1971, Astrophysical Journal, 166, 605, DOI: $10.1086 / 150986$

Zasche, P., Wolf, M., Hartkopf, W. I., et al., A Catalog of Visual Double and Multiple Stars With Eclipsing Components. 2009, Astronomical Journal, 138, 664, DOI: 10.1088/0004-6256/138/2/664

Zola, S., Gazeas, K., Kreiner, J. M., et al., Physical parameters of components in close binary systems - VII. 2010, Monthly Notices of the RAS, 408, 464, DOI: 10.1111/j.1365-2966.2010.17129.x 